\title{
Criminologie
}

\section{De la police guidée par le renseignement à la complexité des appareils policiers : les limites de l'usage des renseignements dans la conduite des affaires policières}

\section{Frédéric Lemieux}

Volume 38, numéro 2, automne 2005

La police en pièces détachées

URI : https://id.erudit.org/iderudit/012662ar

DOI : https://doi.org/10.7202/012662ar

Aller au sommaire du numéro

Éditeur(s)

Les Presses de l'Université de Montréal

ISSN

0316-0041 (imprimé)

1492-1367 (numérique)

Découvrir la revue

Citer cet article

Lemieux, F. (2005). De la police guidée par le renseignement à la complexité des appareils policiers : les limites de l'usage des renseignements dans la conduite des affaires policières. Criminologie, 38(2), 65-89.

https://doi.org/10.7202/012662ar

\section{Résumé de l'article}

Dans cet article, l'auteur explore les limites que pose la police guidée par le renseignement (intelligence led policing - ILP) pour les organisations policières. Il tente de montrer que la fonction de renseignement criminel ne doit pas être comprise comme un instrument produisant des résultats tangibles dans l'application de la loi, mais bien comme une activité de production de connaissance dont l'objectif ultime est d'assurer l'apprentissage des organisations policières. Sur la base de cet apprentissage, les services de police sont en mesure de concevoir et d'affiner leurs stratégies de lutte contre la criminalité. Néanmoins, le renseignement est contraint par des facteurs organisationnels, structuraux et culturels propres à la complexité des bureaucraties professionnelles. D’une part, les limites inhérentes à l'usage des renseignements dans la conduite des affaires policières montrent à quel point cette activité est difficilement mesurable en ce qui a trait aux résultats quantifiables. D'autre part, pour être utile, le renseignement doit s'appuyer sur une structure organisationnelle favorisant la fluidité des échanges d'informations ainsi qu'une forte culture du renseignement. 


\title{
De la police guidée par le renseignement à la complexité des appareils policiers: les limites de l'usage des renseignements dans la conduite des affaires policières
}

\author{
Frédéric Lemieux \\ Professeur adjoint, École de criminologie \\ Chercheur au CICC (Centre international de criminologie comparée) \\ Université de Montréal \\ frederic.lemieux@umontreal.ca
}

\begin{abstract}
RÉSUMÉ - Dans cet article, l'auteur explore les limites que pose la police guidée par le renseignement (intelligence led policing - ILP) pour les organisations policières. Il tente de montrer que la fonction de renseignement criminel ne doit pas être comprise comme un instrument produisant des résultats tangibles dans l'application de la loi, mais bien comme une activité de production de connaissance dont l'objectif ultime est d'assurer l'apprentissage des organisations policières. Sur la base de cet apprentissage, les services de police sont en mesure de concevoir et d'affiner leurs stratégies de lutte contre la criminalité. Néanmoins, le renseignement est contraint par des facteurs organisationnels, structuraux et culturels propres à la complexité des bureaucraties professionnelles. D'une part, les limites inhérentes à l'usage des renseignements dans la conduite des affaires policières montrent à quel point cette activité est difficilement mesurable en ce qui a trait aux résultats quantifiables. D'autre part, pour être utile, le renseignement doit s'appuyer sur une structure organisationnelle favorisant la fluidité des échanges d'informations ainsi qu'une forte culture du renseignement.
\end{abstract}

ABSTRACT - In this article, the author explores the limits of the intelligence led policing within police organisations. He suggests that the function of the criminal intelligence process cannot be taken as an instrument to achieve perceptible results in enforcing the law but as an activity aiming at the production of knowledge. This outcome of this process must then be channelled into a learning process within those organisations in order to develop and refine their strategies to fight crime. Nevertheless, the criminal intelligence process is still constrained by organisational, structural and cultural factors that are specific to professional bureaucracies and their internal 
complexity. On the one hand, the limits on use of information in the management of police affairs show how difficult it is to assess the intelligence process in terms of measurable outcomes. On the other hand, to be useful, intelligence must rely on an organisational structure supporting the constant sharing of information as well as on an occupational culture that strongly values police knowledge.

\section{INTRODUCTION}

La collecte et l'analyse des informations relatives à la perpétration d'un crime ne sont pas des activités récentes dans l'histoire de la police. Aussi loin que l'on puisse remonter, la collecte d'indices, le recueil des témoignages, l'utilisation d'informateurs et la formulation d'hypothèses ont toujours caractérisé la démarche d'un détective. Cependant, au cours des deux dernières décennies, des considérations économiques, organisationnelles, politiques et technologiques, sont venues formaliser le traitement de l'information au sein des services policiers. L'usage du renseignement criminel $^{1}$ a permis de structurer le travail de la police et de parfaire les connaissances des enquêteurs en orientant leurs investigations vers une modélisation théorique du crime organisé : le modèle de l'entreprise criminelle (Peterson, 1994). L'essor du renseignement criminel fait également suite à une désillusion quant à l'efficacité des méthodes traditionnelles de lutte contre les crimes de droits communs au cours des années 1980, notamment en ce qui a trait à l'incapacité des services de police à anticiper et à comprendre l'importante augmentation des taux de la criminalité de cette époque. Enfin, les nombreux scandales concernant les techniques d'interrogatoire, les échecs de la surveillance des criminels récidivistes et la délinquance prolifique ont également contribué à faire progresser l'Intelligence-led-policing (Audit Commission, 1993; Home Office, 1989 et 1995; Maguire, 2000).

Par ailleurs, les années 1980-90 ont été marquées à la fois par une rationalisation des interventions des pouvoirs publics dans le champ de la sécurité intérieure et par une crise de confiance de la population à l'égard de la police (Home Office, 1995 ; Johnston, 1992 et 1999). Cette conjoncture a favorisé l'émergence d'un nombre considérable d'acteurs non gouvernementaux dans le domaine de la sécurité venant ainsi redéfinir le paradigme de l'action policière (Bayley et Shearing, 2001). Cette

1. Le «renseignement criminel» s'intéresse aux problèmes d'ordre et de sécurité en effectuant des analyses portant sur les crimes et les délinquants de droit commun. 
restructuration s'est notamment traduite par l'instauration d'une nouvelle gouvernance de la sécurité dans laquelle la rentabilité et le rendement sont devenus des paramètres incontournables. En effet, les acteurs institutionnels et sociaux exigent désormais une plus grande efficience des organisations policières accentuant ainsi la pression afin qu'elles réduisent leurs coûts de fonctionnement et la taille de leur administration. Ce contexte de rationalisation devait sonner le glas de la réactivité policière orientant plutôt les services policiers vers une proactivité opérationnelle souscrivant à des indicateurs de performance et à une culture du résultat (HMIC, 1997).

De plus, les services de police ont considérablement investi dans l'informatique et les technologies de surveillance dans le but d'accroître leur efficacité opérationnelle. Cet arsenal technologique a doté la police d'une formidable capacité de recueillir et d'analyser des informations provenant de l'environnement social et des milieux criminels. Dans ce contexte, le rôle de la police s'est considérablement transformé, exigeant aujourd'hui des policiers qu'ils deviennent des travailleurs du savoir (Ericson et Haggerty, 1997). En effet, le travail de la police se voit plus que jamais orienté vers la gestion des informations concernant les phénomènes criminels, les personnes physiques et morales contrevenant à la loi. Ces informations sont structurées sous forme de bases de données utiles pour l'étude des tendances spatio-temporelles de la criminalité, la conduite des enquêtes criminelles, le profilage des délinquants, la planification des interventions, l'évaluation des retombées de l'action policière (Ouimet, 1995). Bien que le croisement des informations recueillies par les agences publiques reste encore soumis à un cadre législatif dicté par des impératifs de protection des renseignements personnels (Klosek, 2000), les cadres normatifs se sont élargis de manière notable depuis les dernières années (Brodeur et al., 2003).

Devant les nouvelles exigences de performance imposées par les autorités politiques et le public, les appareils policiers se sont tournés vers une réponse proactive à l'égard des problèmes criminels (Crawford, 1997). S'inscrivant en marge de la police de proximité et de la police de résolution de problème, le modèle de police guidée par le renseignement préconise une utilisation massive des technologies de l'information et des méthodes d'analyse afin d'identifier avec plus de discernement les cibles criminelles prioritaires (Tilley, 2003). L'adoption d'une telle approche exige des organisations policières qu'elles perfectionnent et actualisent régulièrement leurs connaissances des patterns de la crimi- 
nalité, des réseaux criminels et des délinquants prolifiques. La police guidée par le renseignement vient ajouter une forme supplémentaire d'expertise dans les pratiques policières: l'analyse criminelle. Il s'agit en quelque sorte d'un modèle qui systématise le traitement et organise l'information policière afin de: 1) orienter les décisions et le déploiement des ressources; 2) coordonner l'action policière selon différents niveaux de juridiction; et 3) assurer un apprentissage continu à l'aide de rétroactions sur les résultat opérationnels (John et Maguire, 2003). De plus, les connaissances générées par les techniques d'analyse offrent désormais la possibilité d'étendre l'approche managériale de l'ILP vers une gestion actuarielle de la criminalité. Tout comme le domaine de la justice a été amené dans les années 1990 à développer une capacité prédictive à l'égard des risques et des problèmes de récidives (Ericson et Haggerty, 1997; Marx, 1988), les services de police possèdent désormais la capacité d'effectuer des analyses prospectives portant sur les tendances émergentes de la criminalité. Ainsi, l'évaluation des risques et l'évaluation des menaces font désormais partie du vocabulaire et des activités de la police (Maguire, 2000).

L'application et l'exécution de ces objectifs requièrent un personnel spécialisé, des procédures et des structures flexibles permettant de gérer les flux d'informations pour agir promptement, de façon précise et systématique. De plus, ce modèle présente plusieurs avantages: 1) il offre une flexibilité dans le choix des tactiques; 2) il s'écarte des problèmes éthiques liés à l'utilisation de confessions en privilégiant les écoutes électroniques, les délateurs et les informateurs; et 3) il encourage la collaboration entre les détectives, les policiers en uniforme, les employés civils et les agences non policières (Maguire, 2000).

Au-delà des avantages, l'implantation du modèle de police guidée par le renseignement a mis en lumière plusieurs problèmes éthiques, opérationnels et technologiques (Heaton, 2000; Maguire, 2000; Scheptycki, 2000 et 2004; Tilley, 2003). Toutefois, l'ensemble des travaux portant sur l'usage du renseignement criminel offre seulement une compréhension partielle des limites qui caractérisent le modèle de l'ILP. Les obstacles reliés à l'application d'un modèle policier guidé par le renseignement sont considérables. Ils proviennent à la fois de la nature même du renseignement, de la rationalité des acteurs qui évoluent dans ce domaine, de la structure organisationnelle des services de police et de la culture des organisations policières. Nous proposons donc, dans cet article, d'examiner quelles sont les principales limites reliées à l'ac- 
tivité de renseignement criminel. Certes, l'optique que nous adoptons se veut principalement critique et vise à déconstruire le discours des réformateurs qui croient que l'implantation d'une doctrine se traduit simplement par une reconfiguration partielle des structures déjà existantes. Toutefois, cet article n'a pas pour objectif de clamer l'échec du modèle de police guidée par le renseignement, mais bien d'en illustrer les principales limites afin de contribuer à son perfectionnement.

Ainsi, notre argumentation repose sur plusieurs heures d'observation et près de 150 entrevues (individuelles ou en groupe) qui ont été réalisées dans le cadre de trois projets distincts. Tout d'abord, nous avons organisé, en collaboration avec le Service du renseignement criminel du Québec (SRCQ) au cours de la période 2002-2004, plusieurs groupes de discussions et un séminaire portant sur la conception d'une formation en renseignement criminel. Tout au long de cette opération de terrain, nous avons invité les agents de renseignement, les analystes et les gestionnaires d'unités de renseignement criminel de la police québécoise à s'exprimer sur la nature de leur travail, les compétences et les habiletés requises pour évoluer dans ce domaine. Cette activité nous a permis de définir les besoins en matière de formation et les différentes lacunes liées à la constitution d'un savoir pratique quant à la fonction de renseignement criminel (Lemieux, 2005a). Ensuite, avec la collaboration du ministère de la Sécurité publique du Québec, le SRCQ et l'École nationale de police du Québec, nous avons organisé, en février 2004, un colloque provincial portant sur la gestion collective du renseignement criminel auquel ont participé près de deux cents praticiens. Cet évènement nous a donné l'occasion de déceler un certain nombre de contraintes relatives au partage et à l'échange d'informations entre les acteurs individuels et organisationnels faisant partie de la communauté québécoise du renseignement criminel (SRCQ, 2004). Finalement, au cours de l'automne 2004, nous avons visité une douzaine d'organisations policières dans huit pays afin de procéder à une analyse comparative des normes et des pratiques en vigueur dans le domaine $\mathrm{du}$ renseignement criminel (Lemieux, 2005b). À partir d'entrevues semi structurées réalisées auprès du personnel policier, il nous a été possible de mieux comprendre les cadres normatifs qui balisent la fonction de renseignement criminel, de mettre en perspective les différentes formes d'application du modèle de police guidée par le renseignement et de préciser les contraintes structurelles, culturelles et individuelles qui limitent son implantation. 


\section{1- LE RENSEIGNEMENT CRIMINEL: UNE ACTIVITÉ ORIENTÉE VERS DES RÉSULTATS ?}

Étant donné que l'action policière repose essentiellement sur la détection des crimes et puisque les opérations policières ont une incidence limitée sur la découverte des crimes, le renseignement dépend essentiellement de la collaboration du public. Ce contexte pose inévitablement le problème du signalement des délits et de la faible visibilité des crimes que la police traite en priorité (trafic, fraudes, etc.). Or un des principaux problèmes concernant l'utilisation du renseignement dans les opérations policières réside dans l'évaluation de ses retombées. Bien que la littérature montre que la police guidée par le renseignement revêt un potentiel certain dans la lutte contre des formes particulières de crimes, peu de travaux permettent d'affirmer que cette approche affecte de manière notable l'ensemble de la criminalité, les niveaux de détection et la résolution des enquêtes criminelles (Heaton, 2000; Maguire, 2000). En effet, jusqu'à ce jour, aucune évaluation n'a montré que la police guidée par le renseignement représente un modèle "performant» qui génère des résultats quantifiables. Néanmoins, quelques chercheurs ont observé que l'usage des renseignements augmente les probabilités d'arrestation et de condamnation des délinquants ciblés par la police (Eck, 1983; Maguire et John, 1995; Martin et Sherman, 1986). Bien que le modèle de l'ILP préconise la concentration des efforts et des ressources policières sur des objectifs "prioritaires», les résultats de ce ciblage ne semblent pas infléchir les taux de la criminalité. Pourquoi? Essentiellement parce que le renseignement n'est pas tant destiné à concevoir des résultats quantifiables qu'à générer des connaissances et un apprentissage organisationnel. L'activité de renseignement consiste à traiter l'information dans le but de mieux comprendre l'environnement criminel et ses composantes (groupes, individus, etc.). Elle permet également aux organisations policières de mettre sur pied et d'affiner leurs opérations de prévention ou de répression.

Le renseignement criminel possède des finalités stratégiques et tactiques qui sont à la fois distinctes et complémentaires ${ }^{2}$. Le premier a pour objectif la création de connaissances concernant les particularités d'un

2. La complémentarité entre le renseignement stratégique et tactique est parfois difficile à atteindre en raison de l'asymétrie qui prévaut entre les «intérêts supérieurs » de l'organisation, souvent reliée à la médiatisation de certaines affaires, et les préoccupations quotidiennes des enquêteurs qui sont purement opérationnelles. 
problème de sécurité. Il s'intéresse à la nature d'un phénomène criminel, aux particularités des marchés illicites et aux ressources dont dispose l'organisation policière pour lutter contre la criminalité. Parce que l'environnement criminel croît en complexité et en volatilité, le renseignement stratégique a également pour fonction d'anticiper l'évolution spatiotemporelle des problèmes criminels. De plus, il offre la possibilité d'analyser différentes informations quant à l'évolution de phénomènes socio-démographiques, socio-économiques, technologiques et politiques afin de mieux comprendre comment et jusqu'à quel point la criminalité est susceptible d'être affectée par ces changements. De manière générale, le renseignement stratégique oriente le processus décisionnel en tenant compte des objectifs organisationnels. Il a pour fonction de guider les gestionnaires dans le choix des stratégies de contrôle de la criminalité et des phénomènes criminels prioritaires.

Quant au renseignement tactique, il est essentiellement nominatif et permet de mieux connaître une cible criminelle, et ce, dans une fenêtre de temps rétréci. Plus particulièrement, il s'intéresse aux délinquants, à leurs activités, à leur réseau de contacts, aux capitaux qu'ils détiennent et aux autres informations d'ordre personnel. Afin de mieux comprendre les changements qui surviennent dans les milieux criminels, les organisations policières recourent à des opérations de renseignement ponctuelles (tactiques) à l'aide de filatures, d'écoutes électroniques et d'informateurs. Ces activités de surveillance permettront, par exemple, de mieux connaître l'ampleur, la capacité, les forces et les intentions des groupes d'individus associés au crime organisé. L'analyse des informations recueillies dans le cadre de ces opérations de renseignement permet de soutenir le travail des enquêteurs dans les affaires criminelles complexes (codélinquance, crimes sériels, etc.). Enfin, que ce soit sur le plan stratégique ou tactique, les activités de renseignement criminel portent également sur l'évaluation rigoureuse des répercussions découlant des interventions policières ou de tout autres mesures appliquées par le système judiciaire. Les analyses postopérationnelles ont pour objectif la constitution d'un savoir pratique quant à l'efficacité des méthodes et des procédures utilisées dans la lutte contre la criminalité. Elles représentent un des piliers de l'apprentissage organisationnel.

En somme, le renseignement criminel sous-tend un travail intellectuel qui vise l'accroissement, le renouvellement et l'utilisation des connaissances afin de guider les décisions et orienter les opérations policières. Ainsi, les retombées du renseignement ne doivent pas être perçues 
comme des résultats (outcome) quantifiables mais bien comme des produits (output) qualifiables. Certes, nous pourrions croire que le concours $\mathrm{du}$ renseignement tactique dans les enquêtes offre la possibilité de générer des résultats tangibles tels que des arrestations et des perquisitions. Il semble que ce ne soit pas une constante. En fait, l'utilisation du renseignement tactique ne détermine pas systématiquement les résultats d'une investigation policière, mais constitue un élément qui peut certainement contribuer à son succès. Cette nuance est primordiale puisque le renseignement tactique est reconnu pour son manque de fiabilité, il est constamment altéré par des contingences temporelles, environnementales et individuelles. Ainsi, le recours au renseignement comme moyen de créer des résultats demeure une entreprise hasardeuse en raison de la vision partielle et incertaine qu'il fournit. Toute la complexité de l'activité de renseignement se résume dans la capacité de voir, de reconnaitre, de rapporter une information pertinente et d'assurer une réflexion de qualité permettant à l'organisation ou à ses composantes d'apprendre (Keegan, 2004: 325). À cet égard, notons que la rationalité des acteurs interfere dans le traitement de l'information; l'usage des renseignements déformant ainsi les messages en provenance de l'environnement.

\section{2- L'APPLICATION DU MODĖLE DE POLICE GUIDÉE PAR LE RENSEIGNEMENT ET LA RATIONALITÉ LIMITÉE DES ACTEURS}

Le modèle de police guidée par le renseignement met l'accent sur la résolution de problèmes criminels à l'aide de méthodes d'analyses de l'information. Toutefois, cette approche surestime considérablement la capacité de l'esprit humain à résoudre des problèmes complexes tels que la criminalité et les problèmes sociaux connexes. Cette capacité est particulièrement faible lorsqu'elle est mise en relation avec l'ampleur d'un problème et le volume d'informations nécessaires pour mettre en perspective l'ensemble de ses facettes (Simon, 1987). D'une part, les analystes sont limités par leurs habiletés intellectuelles, leurs habitudes et leurs connaissances faisant en sorte qu'ils ne peuvent intégrer toutes les informations qui leurs sont soumises. Ce cas de figure survient lorsqu'il y a une surabondance d'information et que les analystes disposent de peu de temps pour traiter les informations. D'autre part, les techniques d'analyses prescrites par l'organisation, le niveau d'abstraction des 
doctrines, les failles dans les systèmes d'information et le processus irrégulier de production de renseignements accroissent les risques de distorsions cognitives dans le traitement des données policières ${ }^{3}$. Enfin, la logique informelle qui caractérise le fonctionnement des acteurs met en évidence d'autres interférences qui échappent au contrôle direct des analystes et exercent une influence déterminante sur la capacité de résoudre un problème. D'ailleurs, les normes sociales en vigueur dans un milieu de travail interagissent dans le processus de traitement de l'information, notamment lorsque les valeurs de ces derniers se heurtent à celles de l'organisation (Choo, 1998; Pavy, 2002). Bref, le renseignement n'est pas seulement une source de connaissance, il est également une source de confusion.

De plus, le niveau d'incertitude lié aux changements dans l'environnement ou aux activités de l'organisation vient également influencer la rationalité des analystes puisqu'il génère une absence d'information (Dequech, 2001). Pour résoudre un problème de manière optimale, l'individu doit acquérir une connaissance élargie de la situation à laquelle il est confronté et il doit pouvoir anticiper les conséquences qui sont associées à chaque solution qu'il propose. Or la rationalité individuelle est limitée parce que 1) l'esprit humain peut seulement générer un choix très limité de solutions et 2) les conséquences de ce choix sont difficiles à discerner puisque la connaissance du futur demeure imparfaite et fragmentaire. Compte tenu que la prédiction demeure assujettie à l'incertitude de l'avenir, l'imagination devra pallier le manque d'information contraignant l'analyste à fonder son jugement sur l'anticipation des conséquences à l'aide de scénarios caractérisés par une marge d'erreur souvent élevée (Rutherford, 1994). Dans de telles conditions, le renseignement ne peut qu'apporter une vision partielle de l'évolution de la criminalité ou de l'émergence de nouveaux phénomènes criminels. Les organisations policières doivent faire en sorte que les renseignements rendent compte de la portée limitée des analyses pour assurer une plus grande objectivité du traitement de l'information et une utilisation optimale des connaissances introduites dans le processus décisionnel.

Finalement, insistons sur le fait qu'une solution à un problème découle de rationalités largement influencées par les objectifs organisationnels puisque, ne l'oublions pas, les procédures, les normes et les politiques

3. L'information est structurée par des procédés organisationnels qui retardent son accessibilité et la rend disponible de manière séquentielle ou périodique. 
constituent le cadre de référence des employés. Dans un contexte d'incertitude, les individus choisiront non pas la solution «optimale», mais celle qui souscrit à la marge budgétaire, aux intérêts du groupe dominant et à un faible niveau de responsabilité pour celui qui doit prendre la décision (Cohen et Olsen, 1975). Ainsi, peu importe la qualité du renseignement, des «intérêts supérieurs » viendront minimiser ou favoriser sa portée effective dans la décision finale. Cette confrontation entre les objectifs organisationnels et le renseignement invalidera d'entrée de jeu les scénarios envisagés, influençant inévitablement les conséquences futures des solutions retenues. En somme, l'activité de renseignement s'expose à une utilisation erratique puisque les résultats qu'elle génère sont imparfaits pour cinq raisons : 1) l'inaccessibilité de certaines informations ; 2) l'incapacité d'intégrer la masse d'informations dans le processus analytique ; 3) l'impossibilité d'envisager toutes les conséquences des choix retenus ; 4) la divergence entre les objectifs organisationnels et la solution optimale; et 5) l'influence du renseignement sur le processus décisionnel demeure limitée en raison de la logique informelle des acteurs. Dans de telles circonstances, il n'est pas étonnant de constater que les mesures proactives «guidées» par le renseignement procurent des solutions dont les résultats sont difficilement mesurables.

\section{3- STRUCTURER LE RENSEIGNEMENT : INCONGRUENCE DES PARAMÈTRES ORGANISATIONNELS?}

Au cours de la seconde moitié du $\mathrm{XX}^{\mathrm{e}}$ siècle, la doctrine dominante des appareils policiers s'est considérablement transformée. Les services de police sont passés d'un système fermé à celui d'un système ouvert, tout du moins en théorie. Portant une attention croissante à leur environnement, les organisations policières ont entrepris plusieurs changements dans leur approche, dont la proactivité de leurs activités. Parmi les conséquences les plus immédiates de l'adoption d'un modèle reposant sur l'usage du renseignement, on observe des transformations structurelles à la fois à l'intérieur et à l'extérieur des organisations. À l'interne, on note la création d'unités de renseignement, mais leur agrégation à partir de la structure policière déjà existante a été la source de plusieurs échecs. Selon Maguire et John (1995), ces échecs s'expliquent en partie par l'incapacité et le manque de volonté des services de police de rompre avec le modèle réactif. Ainsi, selon les auteurs, les opérations guidées 
par le renseignement fonctionnent seulement si la structure policière permet de le faire, sans tenir compte de la pression exercée par une demande réactive. De plus, selon Tilley (2003), l'implication permanente des policiers dans le modèle de la police guidée par le renseignement pose un problème majeur concernant le temps réellement consacré à la réalisation d'opérations policières et la capacité de maintenir un flux continu d'analyses et d'information. À l'externe, les organisations policières se sont également tournées vers des partenariats afin d'accroître leur «sociabilité» avec d'autres acteurs organisationnels incluant des institutions publiques, parapubliques et privées (Ericson et Haggerty, 1997). Pour assurer la réussite des pratiques de renseignement, les organisations policières ont misé sur la multilatéralisation des échanges d'information avec d'autres agences chargées de l'application de la loi. Elles ont déployé des mécanismes de partage d'informations et de transfert de connaissances qui prennent forme à l'intérieur d'équipes intégrées ou d'opérations conjointes, encadrées par des protocoles officiels de coopération.

Comme l'ensemble des pratiques policières, le renseignement criminel est contraint par des contingences structurelles et organisationnelles liées au modèle bureaucratique des appareils policiers. En nous inspirant des travaux de Langworthy (1986), nous retenons sept caractéristiques organisationnelles qui semblent déterminantes dans la définition d'une structure effective pour le renseignement, soit : la différenciation fonctionnelle, la différenciation occupationnelle, la différenciation spatiale, la différenciation verticale ainsi que le degré de centralisation, de formalisation et l'intensité de l'activité de renseignement. Tout d'abord, la différenciation fonctionnelle réfere à la division d'une tâche entre plusieurs unités distinctes. Du point de vue du renseignement criminel, on remarque que plusieurs organisations policières ont privilégié une structure différenciée, c'est-à-dire que la production de renseignement (notamment au niveau tactique) est réalisée à l'intérieur des brigades spécialisées d'investigation. Bien que la déconcentration des activités de renseignement tactique offre l'avantage de situer l'analyste au cœur des opérations et de favoriser la création de liens de confiance avec les enquêteurs, il n'en demeure pas moins que cette approche provoque également l'isolement ou la subordination des analystes à un petit groupe d'enquêteurs influents. Ainsi, dans une structure différenciée, le traitement de l'information risque d'être réalisé de manière insulaire en se concentrant dans 
les unités spécialisées ou d'être intégré à un mode féodal de gestion de l'information. Ce contexte mine l'adéquation entre les objectifs opérationnels et les priorités organisationnelles.

En règle générale, on retrouve une faible différenciation occupationnelle dans les unités de renseignement. On observe que les organisations policières n'ont pas vraiment recours à des expertises reconnues dans le traitement de l'information. En général, le degré de spécialisation découle du niveau de formation et de l'expérience relative à la réalisation d'un travail. Elle résulte également d'un partage des connaissances et de la communication entre les experts assignés à un travail particulier. Bien que la tendance des dernières années soit d'augmenter le degré de spécialisation des policiers (Skogan et Frydl, 2004), les unités de renseignement criminel sont caractérisées par de profondes lacunes quant à la reconnaissance des expertises provoquant parfois des clivages entre les groupes professionnels qui les constituent ${ }^{4}$. Une faible différenciation occupationnelle émane du flou entourant la définition des tâches ou des spécialités. Elle occasionne une duplication des activités professionnelles et un empiètement des expertises qui entraîne l'incursion dans des chasses gardées. Dans un tel cas de figure, la différenciation occupationnelle suscite de l'incompréhension, de la méfiance et de la rivalité entre les groupes de professionnels et, par conséquent, favorise l'instauration de mécanismes de rétention d'information. Enfin, il semble que les syndicats policiers freinent considérablement la spécialisation des organisations policières en tentant de monnayer (parfois à grands frais) la formation et l'apprentissage de leurs membres. Dans certain cas, ils verrouillent toutes possibilités d'accroître la qualité des ressources humaines en refusant au personnel civil d'occuper des postes spécialisés au sein des unités de renseignement criminel. Cette réaction des syndicats génère des conflits internes qui trouvent leurs solutions dans des compromis administratifs dont les répercussions à moyen terme pervertissent le modèle de l'ILP et ces principaux objectifs.

En troisième lieu, on retrouve la différenciation spatiale, c'est-à-dire l'étendue ou la concentration géographique des activités de renseignement. Afin de s'attaquer à des problèmes de sécurité se distribuant différemment

4. L'introduction de personnels civils possédant un niveau de scolarité universitaire élevé mais n'ayant aucune expérience policière, la présence de policiers de carrière cumulant plusieurs années d'expérience mais possédant un faible niveau de scolarité et la présence de personnel administratif ne possédant ni expérience policière ni diplôme universitaire représente une différenciation occupationnelle axée sur le statut plutôt que l'expertise. 
en fonction des caractéristiques du territoire et des communautés, le modèle de police guidée par le renseignement repose généralement sur un programme de renseignement orienté vers un nivellement géographique (local, régional et national) des phénomènes criminels. La différenciation spatiale des activités de renseignement revêt une importance considérable puisqu'elle influence la capacité organisationnelle de créer des liens avec les acteurs clés présents dans l'environnement, de recueillir et de faire circuler les informations qui répondent aux préoccupations des principaux destinataires à chacun des niveaux géographiques ou juridictionnels. Or cette différenciation spatiale génère un paradoxe intéressant puisqu'en voulant abattre les frontières juridictionnelles entre les services policiers et instaurer une multilatéralisation des échanges d'informations entre eux, l'ILP structure tout de même le traitement de l'information en fonction d'une hiérarchisation géographique des préoccupations ou des problèmes criminels. L'application d'un tel modèle en Angleterre a mis en évidence les limites opérationnelles de cette conceptualisation puisque l'uniformisation des problèmes de sécurité selon des niveaux géographiques préétablis ne correspond pas nécessairement à la structure des activités criminelles, des marchés illicites et des organisations criminelles (Sheptycki, 2004).

Le modèle de police guidée par le renseignement sous-tend également une structuration des flux d'informations qui se heurte à la profondeur de la hiérarchie. Bien que la structure hiérarchique assure un rôle de filtre afin d'éviter que les niveaux inférieurs ou supérieurs soient submergés par une masse d'informations, il n'en demeure pas moins que la forte différenciation verticale que sous-tend les bureaucraties policières est néfaste à la fluidité des échanges d'informations. En effet, une hiérarchie trop étendue entraîne une variété de problèmes tels qu'une communication défaillante et une faible rétroaction concernant la pertinence ou la qualité des informations transmises. Elle favorise également la concentration des informations à l'intérieur de divisions ou d'unités opérationnelles spécialisées. En revanche, nous observons que l'introduction des technologies de l'information vient briser la segmentation de l'information. Elles offrent une structure qui permet les collaborations entre les différents secteurs organisationnels permettant de contourner les voies hiérarchiques traditionnelles. Ainsi, la mise en place de passerelles et de réseaux virtuels a contribué à la substitution de la fragmentation sectorielle au profit d'une cohésion des échanges d'informations. Néanmoins, soulignons que la technologie maintient les différences de statut et ne 
diminue en rien les problèmes de communication associés à la distance culturelle des acteurs.

De plus, le modèle de police guidée par le renseignement requiert un processus décisionnel flexible dans une structure décentralisée. Il permet aux unités de renseignement d'adapter leurs activités aux variations qui surviennent dans l'environnement criminel. Une structure décentralisée offre également la possibilité que l'autorité soit déléguée aux professionnels qui ont pour tâche de gérer et de traiter les flux d'informations. Bien que les événements du 11 septembre aient entraîné la centralisation des appareils policiers, on observe depuis quelques années une tendance à la décentralisation des activités policières, notamment dans le domaine du renseignement et des enquêtes criminelles. De plus, la réalisation d'investigations conjointes et le nombre d'équipes intégrées gagnent en popularité. Ces circuits de communication deviennent des médias incontournables pour la circulation de l'information, le partage des connaissances et le maintien des interactions sociales entre les agences d'application de la loi (Ericson et Haggerty, 1997 : 45). Jusqu'à maintenant, elles ont su montrer leur efficacité dans l'exécution de «missions spéciales» exigeant un niveau élevé d'innovation dans la conduite des investigations et dans le traitement de l'information.

Du point de vue du renseignement, une telle décentralisation structurelle fait en sorte que l'interaction entre les agences policières devient l'un des principaux outils de production d'information dans la lutte contre le crime organisé. Néanmoins, la conception d'une telle structure sous-tend un processus décisionnel flexible qui repose sur deux conditions essentielles: 1) les rapports qui se créent entre les acteurs doivent être caractérisés par des échanges soutenus, une production d'information continue et une structure permanente; 2) la structure des rapports entre les acteurs doit découler d'une interdépendance et d'une coexistence reposant sur la réciprocité ainsi que sur la stabilité des relations. En effet, des travaux récents ont montré que la composition, la taille du groupe, la familiarité entre les membres et le processus de communication jouent un rôle prééminent dans la résolution des problèmes et le processus décisionnel (Wastell et al., 2004). Toutefois, ces escouades mixtes fournissent des renseignements qui sont seulement utiles pour leurs propres objectifs et peu de ressources, voir aucune, ne sont consacrées à l'intégration des renseignements afin d'assurer un processus d'apprentissage continu pour les organisations policières qui participent au fonctionnement de ces structures. De plus, ces structures sont suscep- 
tibles d'engendrer des tensions inhérentes à la complexité des rapports entre les agences et leurs représentants si les conditions mentionnées précédemment ne sont pas respectées. En effet, la création d'alliances informelles, la divergence des priorités et des agendas des acteurs viennent accroître le degré d'incertitude quant à l'atteinte des objectifs communs minant ainsi les efforts collectifs de production de renseignement.

Par ailleurs, les organisations policières sont reconnues pour leur degré élevé de formalisme, c'est-à-dire un encadrement rigide dans lequel les opérations et les employés sont contraints par des règles procédurales, des politiques internes et des lois. L'exécution d'une tâche exige un certain niveau de formalisation afin de rendre explicite des connaissances tacites reliées à une occupation. Le modèle de police guidée par le renseignement n'échappe pas à cette réalité. La formalisation prend forme, entre autre, dans l'instauration d'une doctrine qui structure l'activité de renseignement selon une démarche empruntée au domaine scientifique afin d'encadrer le processus de traitement de l'information (Godfrey et Harris, 1971)5. On remarque ainsi un degré élevé de formalisme dans les méthodes de travail, notamment en ce qui a trait à la planification des opérations de renseignement, le profilage des problèmes et des cibles criminelles, l'établissement d'indicateurs de performance. Cette formalisation vient modifier les exigences de spécialisation, de compétence et de qualification du personnel policier.

Toutefois, on observe que le traitement des informations s'effectue par un processus formel de codification qui engendre parfois une compréhension erronée ou limitée d'un événement criminel altérant ainsi la qualité des renseignements produits (Manning, 1989). De plus, la complexité des procédures peut restreindre considérablement l'activité $\mathrm{du}$ renseignement puisqu'elle suscite des frictions entre l'obligation de moyens des unités de renseignement et l'obligation de résultats des escouades spécialisées. Ainsi, la pression exercée par les unités opérationnelles ou par l'état-major conjuguée à la volonté de ne pas décevoir des destinataires qui reconnaissent à peine l'utilité du renseignement, font en

5. Le processus comprend plus ou moins les 7 étapes suivantes: 1) Demande de renseignements ou besoin de renseignements; 2) Planification de la cueillette des informations; 3) Collecte des informations; 4) Appréciation et encodage des informations; 5) Analyse des informations (tactiques ou stratégiques); 6) Diffusion des renseignements; 7) Évaluation des retombées de l'opération de renseignement (répercussions ou utilité des renseignements); Selon l'évaluation, le processus pourra être réinitialisé pour répondre à des besoins supplémentaires ou tout simplement pour démarrer une nouvelle opération de renseignement. 
sorte que le processus de traitement de l'information est parfois tronqué. Bien que les membres des unités de renseignement considèrent l'évaluation des retombées d'une opération de renseignement comme une étape indispensable à la procédure de traitement de l'information, celle-ci est rarement réalisée faute de temps et de ressource. Enfin, mentionnons que l'activité de renseignement est soumise à différents règlements concernant l'accès à l'information et la protection des renseignements personnels qui encadrent les échanges entre les agences chargées de l'application de la loi. Le partage des informations est également limité par les politiques internes de sécurité des organisations policières en raison de la diversité des critères utilisés pour déterminer le niveau de confidentialité accordé à un renseignement. L'étendu et l'opacité du cadre normatif qui régule les échanges d'information engendrent un excès de prudence et même de la méfiance affectant inévitablement les flux d'information.

Finalement, l'intensité de l'activité de renseignement criminel, c'està-dire la proportion de ressources organisationnelles dévolue à cette tâche, est directement reliée aux chances de succès de police guidée par le renseignement. Selon Maguire (2000: 332), bien que les gestionnaires de services de police et les décideurs politiques pressent les policiers de s'orienter vers un travail plus proactif, il en résulte néanmoins un faible investissement en ressources humaines et financières dans les activités de renseignement. Compte tenu que l'efficacité du modèle de l'ILP repose sur l'intensité de l'activité de renseignement, la taille des corps de police pose inévitablement le problème de la disponibilité des moyens. En Amérique du Nord, l'éclatement des appareils policiers selon des niveaux de juridiction (local, provincial, fédéral, etc.) contribue à maintenir des services de police de tailles très variées. Cette situation génère des prédispositions organisationnelles asymétriques à intégrer le modèle de police guidée par le renseignement puisque, toutes choses étant égales par ailleurs, la taille des services de police est susceptible de refléter la propension à mener des opérations de renseignement de manière soutenue. Ainsi, la hiérarchisation des flux d'information proposée par le modèle de l'ILP devient, d'entrée de jeu, déficiente. En effet, les risques de déséquilibre dans les échanges d'information sont considérables en raison de la faible capacité des services de police locaux de mettre en place une activité de renseignement criminel suffisamment intense pour alimenter les autres niveaux juridictionnels supérieurs. 


\section{4- UN COMPROMIS ENTRE LA CULTURE POLICIÈRE ET LA CULTURE DU RENSEIGNEMENT ?}

Contrairement aux organisations de type commercial, les organismes publics sont caractérisés par un conformisme institutionnel qu'ils acceptent comme étant la vérité et par un environnement politique qui les obligent à rendre des comptes (Crank, 2003; Mastrofski et Uchida, 1993). De plus, les services de police ne sont pas tenus d'innover et d'améliorer la qualité de leurs services afin de résister à un environnement compétitif qui pourrait les absorber. Ces constats laissent à penser que les organisations policières peuvent difficilement instaurer, et surtout maintenir, une doctrine qui met l'accent sur l'utilitarisme et l'instrumentalisme propres aux entreprises commerciales. Ainsi, le modèle de police guidée par le renseignement, fondé sur une doctrine corporative, semble contrevenir à la culture bureaucratique des appareils policiers. Néanmoins, si l'on considère que les services de police opèrent dans un environnement institutionnel compétitif et qu'ils subissent constamment des pressions afin de fournir des résultats, il est envisageable qu'une culture de performance émerge ${ }^{6}$. D'ailleurs, ce sont justement les organisations qui subissent de telles pressions de l'environnement qui favorisent une communication interne fluide et une forte implication des membres dans le partage des connaissances (Aiken et Hage, 1968).

Bien que le modèle de police guidée par le renseignement vienne rompre avec la conception traditionnelle du travail policier, il souscrit en plusieurs points à la culture policière - plus que le modèle de police communautaire à tout le moins. En effet, l'ILP suggère que la mission de la police soit essentiellement guidée par l'application de la loi et que les besoins d'intervention soient principalement déterminés par les organisations policières, non par la communauté. Dans ce contexte, le discours des policiers porte sur des considérations légales et ils se montrent «uniquement» perméables aux agences chargées de l'application de la loi en raison de la confidentialité entourant la gestion des renseignements. Les priorités du gouvernement deviennent la seule source de légitimité de l'action policière et les opérations entreprises par la police sont réalisées afin de renforcer cette légitimité auprès des autorités politiques. Enfin, les tactiques privilégiées sont les interventions policières à

6. Mentionnons à cet égard que la Police fédérale australienne et la Police de Singapour sont membres de la Chambre de commerce de leur pays respectif et ont gagné plusieurs prix pour leurs performances. 
grande échelle, qui génèrent des arrestations massives, des perquisitions volumineuses et la dislocation des réseaux de délinquants (Tilley, 2003: 325-326).

Toutefois, selon Maguire (2000: 332), il semble que ce discours n'ait pas réussi à pénétrer tous les secteurs d'activités de la police, notamment la gendarmerie et certains services d'enquête réputés pour leur fermeture. Une des raisons qui expliquent cette résistance réside dans le choix des porte-parole, c'est-à-dire les cadres intermédiaires et supérieurs. En effet, la culture occupationnelle de ces derniers les oppose diamétralement aux policiers qui sont assignés aux unités opérationnelles. Cette différence se manifeste également dans leur représentation du renseignement puisque bon nombre de gestionnaires considèrent que «le renseignement, c'est le pouvoir». Cette philosophie est justement contraire à la culture du renseignement puisqu'elle suggère que le gestionnaire, tel un souverain, exerce un contrôle sur le flux de l'information dans le but de moduler le processus décisionnel. Or si l'information devient une source de pouvoir, de promotion ou de gloire, le ou les détenteurs trouveront difficile de la partager et favoriseront plutôt sa concentration. C'est d'ailleurs dans cette logique que s'enferment les relations entre les unités opérationnelles et le reste de l'organisation policière (Brown, 1981; Manning, 1977 ; Reuss-Iianni, 1983). Mentionnons également le dogmatisme du personnel cadre qui occupe une position privilégiée et qui a le pouvoir de discréditer les idées provenant du personnel hiérarchiquement inférieur. Ce manque d'ouverture a pour conséquence de neutraliser tout processus de réflexion quant à l'évolution des menaces et aux mesures qui doivent être déployées pour les contrer.

Soulignons par ailleurs que la fonction de renseignement est caractérisée par une activité irrégulière qui s'explique, entre autres, par un fort mouvement de personnel. Les policiers ont accès à une variété de postes dans l'organisation faisant en sorte qu'ils poursuivent rarement une carrière dans le domaine du renseignement, et ce, en raison de leurs ambitions professionnelles ou des règles administratives qui encadrent les conditions d'affectation. D'une part, nous avons observé que les policiers utilisent les unités de renseignement comme tremplin professionnel afin d'atteindre des positions élevées dans les unités spécialisées réputées ou au sein de l'état-major. D'autre part, nous avons également remarqué que certaines conventions syndicales imposent un renouvellement cyclique du personnel des unités de renseignement. Bien que l'objectif 
est d'éviter la déqualification du personnel policier et la volonté d'introduire des ressources ayant de nouvelles idées, on observe, par ailleurs, qu'il s'agit d'une manœuvre visant à aménager une porte de sortie au personnel qui se destine à la retraite et à ceux qui ont reçu un avis de réduction de tâche pour des raisons médicales (blessure, épuisement psychologique, etc.). Cette réalité affecte directement la mémoire organisationnelle en provoquant une amnésie partielle à l'égard des mécanismes informels d'échange d'information et des changements environnementaux (Wang et Ahmed, 2003). De plus, si l'on considère que l'autonomie d'un analyste est effective après quelques années de formation et d'expérience, force est d'admettre que ces pratiques sont néfastes à la stabilité de la production de renseignements et à l'apprentissage de l'organisation policière.

Par ailleurs, la différenciation des statuts provoquant un entrechoquement des croyances professionnelles constitue la principale source de conflits au sein des unités de renseignement. Bien qu'elles aient tendance à s'amenuiser avec le temps, les tensions les plus palpables surviennent entre les personnels civil et policier. La méconnaissance de la fonction d'analyste, les faibles possibilités de promotion des employés civils et le fait que plusieurs policiers croient qu'ils sont les seuls à saisir la subtilité du travail d'enquête génèrent une double frustration chez les membres civils. D'un côté, le traitement différentiel qu'ils reçoivent les place en marge du milieu policier et, de l'autre, le fait de ne pas disposer d'une reconnaissance professionnelle contribue à une sous-utilisation de leurs capacités intellectuelles. À court terme, cette situation entraîne une polarisation entre les effectifs policiers et civils altérant la fluidité des communications dans les unités de renseignement ${ }^{7}$. Afin d'atteindre une diffusion optimale du modèle de police guidée par le renseignement, les organisations policières seront contraintes de revoir leur conception traditionnelle des expertises à l'intérieur des unités chargées du renseignement. La gestion des flux d'information ne doit pas être assujettie aux statuts ou aux rangs hiérarchiques. Elle doit plutôt être l'apanage des experts chargés de l'analyse des informations, de l'organisation de l'information et des systèmes d'information. Bien que plusieurs organisations policières aient différencié le travail de l'analyste de celui de

7. Cependant, avec le temps, on observe une atténuation des tensions si l'organisation favorise la création de liens informels en dehors des heures de travail à l'aide d'activités socialisantes (récréatives ou formatives). 
l'agent de renseignement, ces deux domaines d'expertises demeurent néanmoins peu reconnus puisqu'ils sont souvent cumulés ou interchangés pour des raisons de rentabilité.

Plus encore, les relations entre les membres d'unités de renseignement et ceux des unités opérationnelles sont également difficiles en raison d'un chevauchement apparent des tâches. Cette superposition occupationnelle attise parfois la méfiance des enquêteurs qui ne voient pas d'un bon œil que leur travail soit scruté, commenté et parfois même parachevé par des analystes. Les similitudes entre la fonction d'analyse et d'investigation font en sorte que les enquêteurs sont poussés dans leur retranchement et adoptent une attitude défensive afin de préserver les prérogatives de leur statut. Enfin, des tensions existent également entre les analystes stratégiques et les membres des unités opérationnelles, ces derniers étant réticents à partager leurs informations puisqu'ils n'entrevoient pas la réciprocité de leur geste. L'essence même de l'analyse stratégique semble échapper aux unités opérationnelles qui sont exclusivement orientées vers l'action. Leurs relations sont rarement fondées sur la réciprocité et la mutualité dans les échanges d'informations altérant ainsi les liens de confiance.

Finalement, le modèle de police guidée par le renseignement préconise une multilatéralisation des échanges d'information afin d'accroître l'efficacité de l'action policière. Les unités mixtes ou équipes intégrées offrent la possibilité d'accroître une responsabilité mutuelle entre les individus et les organisations policières. Elles favorisent également une forte spécialisation qui augmente l'interdépendance entre les policiers et stimule le renforcement de rapports sociaux structurés et fréquents (Zolin et al., 2003 : 3 ; Wastell et al., 2004). A contrario, une forte mixité culturelle peut également réduire la portée des ententes et des protocoles de coopération entre les partenaires en raison des divergences culturelles et de l'asymétrie structurelle des agences policières (Medd, 2001). À cet égard, mentionnons que des travaux portant sur la gestion de l'information ont montré qu'il existe une relation négative entre la confiance interpersonnelle et la distance culturelle, l'augmentation de cette dernière inhibe la confiance (Luo, 2002). Soulignons également que le manque de standardisation dans la terminologie ainsi que l'absence d'harmonisation dans les pratiques représentent des barrières considérables à la collaboration policière. Enfin, rappelons que les unités mixtes ont été déployées pour lutter contre des phénomènes criminels majeurs (crime organisé) et subissent, par conséquent, de fortes pressions organisation- 
nelle, médiatique et politique pour fournir des résultats. Bien que le fait d'évoluer dans un environnement «aversif» augmente la capacité informationnelle des équipes intégrées, cette situation provoque également une accélération des processus de production d'information et une diminution du degré de formalisation des échanges. Il en résulte une production élevée de connaissances tacites ${ }^{8}$ et une réduction réelle de l'apprentissage organisationnel.

En somme, l'approche managériale que sous-tend le modèle de police guidée par le renseignement insiste fortement sur les résultats sans toutefois assoir réellement les bases d'une culture du renseignement. Or, selon Choo (1998: 10), le quotient intellectuel d'une organisation se mesure par la capacité d'accéder et de reconnaitre l'information utile à son fonctionnement; la capacité d'intégrer et de communiquer les informations tant à l'interne qu'à l'externe; la capacité d'interpréter et de comprendre les signaux provenant de l'environnement afin d'en tirer un apprentissage. La mise en place d'une organisation «intelligente» exige un climat qui favorise l'apprentissage, la conception de processus de traitement de l'information et de procédures qui font la promotion de la production de connaissances et de leurs utilisations (Choo, 2001). Ainsi, les organisations policières doivent éviter les situations où les informations sont perdues, où les individus sont écoutés seulement s'ils viennent eux-mêmes transmettre leurs informations, où la responsabilité de transmettre l'information est inexistante, où le partage de l'information est autorisé mais souvent négligé, où l'organisation est trop indulgente envers ceux qui omettent de communiquer l'information et où les nouvelles idées représentent une menace (Choo, 2001: 55). En général, ces situations témoignent d'une attitude hostile au partage de l'information, d'une faible capacité de rétroaction sur la valeur des renseignements transmis, d'un niveau élevé de bureaucratie et d'un faible intérêt pour l'innovation. Dans de telles conditions, l'organisation ne fonde pas ses stratégies d'action sur la connaissance, mais bien sur les mythes et les valeurs qui caractérisent sa culture (McGill et Slocum, 1994). Le défi que représente l'intégration d'une police guidée par le renseignement au sein des organisations policières tient au fait qu'elles doivent changer

8. Les connaissances tacites sont utilisées par les membres d'une organisation afin de réaliser leur travail et donner un sens à ce qu'ils font. Elles ne font pas l'objet d'une codification systématique, elles sont difficiles à communiquer et elles sont à la base du jugement intuitif. 
plusieurs traits de leur culture afin de stimuler la confiance interindividuelle dans le partage de l'information, d'intensifier les rétroactions sur l'utilité des renseignements produits et d'encourager les innovations.

\section{CONCLUSION}

Afin d'accroître leur efficacité face à un environnement où les changements environnementaux modulent les niveaux de la criminalité et influencent l'émergence de nouveaux phénomènes criminels, les organisations policières tentent d'adopter une approche proactive dans la lutte contre la criminalité en s'appuyant sur l'usage du renseignement. Le modèle de police guidée par le renseignement mise sur une meilleure compréhension de l'environnement criminel afin de saisir les opportunités d'intervention et de choisir les stratégies ou les tactiques les plus efficaces pour agir. Ces dernières sont elles-mêmes issues d'un processus d'apprentissage qui résulte de l'évaluation méthodique des opérations et de leurs répercussions sur l'environnement. L'implantation de cette doctrine a considérablement amélioré la capacité informationnelle des appareils policiers. Néanmoins, la production de connaissances dans une organisation résulte d'influences interdépendantes entre la gestion des obstacles qui se dressent dans la structure organisationnelle et les limitations individuelles. La gestion des connaissances dépasse maintenant le simple stockage des données, elle comprend un ensemble complexe d'interactions entre des facteurs sociaux, les systèmes de communication et la complexité organisationnelle (Beesley, 2004 : 7273). De plus, le renseignement criminel demeure une activité sensible aux valeurs, aux croyances et aux rationalités des individus qui évoluent dans ce domaine.

Tout comme la plupart des organisations, les services policiers sont en mesure d'adapter leurs actions sur le terrain, mais éprouvent des difficultés notables à remettre en cause les valeurs qui les guident lorsque surviennent des changements dans l'environnement. En se réfugiant dans une conception étroite du milieu criminel, les organisations policières risquent de perdre toute capacité d'apprendre et d'innover. Pour juger de la réussite d'un modèle tel que la police guidée par le renseignement, l'attention ne doit pas être portée sur le nombre d'arrestations, de perquisitions et de condamnations, mais bien à la qualité des connaissances générées et à la capacité d'apprendre à partir de celles-ci. Le propos de 
cet article n'a donc pas seulement pour objectif de démontrer que le modèle de l'ILP fait face à de nombreux obstacles, il se veut une réflexion sur la capacité et la volonté réelle des organisations policières à s'engager dans un processus continu de création de connaissances et d'apprentissages qui améliorera l'efficacité de leurs actions. Pour en arriver à une implantation réussie de ce modèle, les réformes doivent dépasser les simples changements structuraux et générer les conditions nécessaires à son intégration, soit une culture du renseignement, une spécialisation accrue du personnel et un aplanissement des contraintes organisationnelles.

\section{Références}

Aiken M. \& Hage, J. (1968). Organisational Interdependence and Intraorganisational Structure. American Sociological Review, 33, 912-930.

Audit Commission (1993). Helping with enquiries. Tackling crime effectively. London: HMSO.

Bayley, D. \& Shearing, C. (2001). The new structure of policing: description, conceptualisation, and research agenda. Washington: National Institute of Justice.

Beesley, L. (2004). Multi-level complexity in the management of knowledge networks. Journal of Knowledge Management, 8 (3), 71-88.

Brodeur, J.-P., Gill, P. \& Tölborg, D. (2003). Democracy, law, and security: internal security services in contemporary Europe. Burlington: Ashgate.

Brown, M. K. (1981). Working the street: Police discretion and the dilemmas of reform. Troy: Russell Sage Foundation.

Choo, C. W. (1998). The knowing organisation: How organizations use information to construct meaning, create knowledge, and make decisions. Oxford: Oxford University Press.

Choo, C. W. (2001). Information management for the intelligent organization. Medford, $\mathrm{NJ}$ : Information today.

Cohen, J. \& Olsen, J. (1975). The uncertainty of the past: organizational learning under uncertainty. European Journal of political research, 3, 147-171.

Crank, J. P. (2003). Institutional theory of police: a review of the state of the art. Policing: An international journal of police strategies and management, 26 (2), 186-207.

Crawford, A. (1997). The local governance of crime: appeals to community and partnerships. London: Oxford University Press.

Dequech, D. (2001). Bounded rationality, institutions and uncertainty. Journal of Economic Issues, 35 (4), 911-929.

Eck, J. E. (1983). Solving crimes: the investigation of burglary and robbery. Washington: Police Executive Research Forum. 
Ericson, R. V. (1994). The Royal Commission on criminal justice system surveillance. In M. McConville \& L. Bridges (ed.), Criminal justice in crisis (113140). Aldershot: Edward Elgar.

Ericson, R. V. \& Haggerty, K. D. (1997). Policing the risk society. Oxford: Clarendon Press.

Godfrey, E. D. \& Harris, D. R. (1971). The basic elements of intelligence. Washington: Law enforcement assistance administration.

Heaton, R. (2000). The prospects for intelligence-led policing: some historical and quantitative considerations. Policing and Society, 9, 337-355.

HMIC (1997). Policing with intelligence. London: Her Majesty's Inspectorate of Constabulary.

Home Office, Statistical Bulletin (1989). Criminal and custodial careers of those born in 1953, 1958 and 1963. London: Home Office.

Home Office, Statistical Bulletin (1995). Criminal careers of those born between 1953 and 1973. London: Home Office.

John, T. \& Maguire, M. (2003). Rolling out the National Intelligence Model: Key Challenges. In K. Bullock \& N. Tilley (ed.), Crime Reduction and ProblemOriented Policing (38-68). Cullompton Devon: Willan Publisher.

Johnston, L. (1992). British policing in the nineties: free market and strong state? International Criminal Justice Review, 2, 1-18.

Johnston, L. (1999). Private policing in context. European Journal on Criminal Policy and Research, 7 (2), 175-96.

Keegan, J. (2004). Intelligence in war. The value and limitations of what the military can learn about the enemy. New York: Random House.

Kloseck, J. (2000). Data privacy in the information age. New York: Quorum Books.

Langworthy, R. H. (1986). The structure of police organizations. New York: Praeger.

Lemieux, F. (2005a). Le développement d'un réseau de renseignements policiers au Québec: de la formation à l'imputabilité. Revue canadienne de criminologie et de justice pénale, 47 (1), 45-62.

Lemieux, F. (2005b). Normes et pratiques en matière de renseignements criminels: une comparaison internationale. Montréal: Rapport de recherche, Université de Montréal.

Luo, Y. (2002). Building trust in cross-cultural collaborations : toward a contingency perspective. Journal of management, 28 (5), 669-694.

Maguire, M. (2000). Policing by risks and targets : Some dimensions and implications of intelligence control. Policing and Society, 9, 315-336.

Maguire, M. \& John, T. (1995). Intelligence, surveillance and informants : Integrated approaches. London: Home Office Research Group.

Manning, P. K. (1977). Police Work: The Social Organization of Policing. Cambridge: The MIT Press.

Manning, P. K. (1989). Symbolic communication. Signifying calls and the police response. Boston: MIT Press. 
Martin, S. E. \& Sherman, L. (1986). Selective apprehension : A police strategy for repeat offenders. Criminology, 77 (4), 1159-1182.

Marx, G. T. (1988). Undercover : Police Surveillance in America. Berkley: University of California Press.

Mastrofski, S. \& Uchida, C. (1993). Transforming the Police. Journal of Research in Crime and Delinquency, 30, 330-358.

McGill, M. E. \& Slocum, J. (1994). The Smarter Organization: How to Build a Business that Learns and Adapts to Marketplace Needs. New York: John Wiley and Sons.

Medd, W. (2001). Complexity and partnership. In D. Taylor (ed.), Breaking down barriers: Reviewing partnership practice (29-46). Brighton : Brighton University Press.

Ouimet, M. (1995). Vers une police informée. Revue internationale de criminologie et de police technique, 1, 87-94.

Pavy, G. (2002). La logique de l'informel. Paris: Les Éditions d'organisation.

Peterson, M.-B. (1994). Application in criminal analysis. A source book. Wesport: Greenwood Press.

Reuss-Iianni, E. (1983). Two cultures of policing. New Brunswick: Transaction Books.

Rutherford, M. (1994). Institutions in economics. Cambridge: Cambridge University Press.

Service du renseignement criminel du Québec (2004, février). Pour que l'information devienne renseignement. Acte du colloque tenu à Nicolet, Québec.

Sheptycki, J. (2000). Editorial reflections on surveillance and intelligence-led policing. Policing and Society, 9, 311-314.

Sheptycki, J. (2004). Organizational pathologies in police intelligence: Some contributions to the lexicon of intelligence-led policing. European Journal of Criminology, 1 (3), 307-332.

Simon, H. (1987). Bounded rationality. In J. Eatwell, M. Milgate, \& P. Newman (ed.). The New Palgrave Dictionary of Economics (266-268). London, England: Macmillan.

Skogan, W. \& Frydl, K. (2004). Fairness and Effectiveness in Policing: The Evidence. Washington, DC: The National Academies Press.

Tilley, N. (2003). Community policing, problem-oriented policing and intelligence-led policing. In T. Newburn (ed.), Handbook of policing (311-339). Portland: Willan publishing.

Wang, C. L. \& Ahmed, P. K. (2003). Making Organisational Memory Perform.

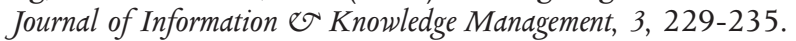

Wastell D., Kawalek, P., Langmead-Jones, P. \& Ormerod, R. (2004). Information systems and partnership in multi-agency networks: an action research project in crime reduction. Information and organization, 14, 189-210.

Zolin R., Hinds, P. J. Fruchter, R. \& Levitt, R. E. (2003). Interpersonal distrust in cross-functional, geographically distributed work: A longitudinal study. Information and organization, 4, 1-26. 